les meilleures conditions pour l'écoulement direct et sans perte de charge de la vapeur à condenser, et elle permet de réduire à la dernière limite l'encombrement occupé.

Avant de quitter ce sujet, il est bon de remarquer que la disposition verticale n'est guère réalisable quavec les principes sur lesquels sont hasées les turbines Curtis : très petit nombre de roues de grande vitesse périphérique et, par suite, de grand diamètre, conduisant à une grande largeur diamétrale et à une faible dimension longitudinale, et permettant de donner aux machines une assise et une stabilité qu'il serait très difficile de réaliser avec les proportions admises pour les turbines genre Parsons ou Zoelly.

Meilleures conditions à réaliser pour l'établissement des turbines $a ̀$ vapeur. - Les turbines à vapeur ne réalisent tous lcurs avantages économiques qu'en marchant à condensation avec un très bon vide.

Alor's qu'un vide de 85 \% suffit très bien aux machines à piston, il ne faut pas réaliser moins de $90 \%$ avec les turbines, et il est même préférable d'atteindre 93 à $94 \%$ D'une manière générale, on aura presque toujours intérê à réaliser un vide aussi élevé que le permettront la température et la quantité d'eau de condensation dont on disposera.

Ce fait se comprend d'ailleurs aisément, puisqu'à l'inverse des machines à piston, les turbines utilisent la détente totale de la vapeur jusqu'à la pression du condenseur (1) ; en outre, la plus grande raréfaction de la vapeur doit conduire à une certaine diminution du frottement des roues motrices dans le fluide ambiant, principalement aux dernier's stages.

En chiffres ronds, on a constaté une réduction dans la consommation de vapeur d'environ $1 \%$ par centième d'atmosphère de diminution de la pression d'échappement, et une augmentation encore plus marquée pour la puissance développée, dans les mêmes conditions d'admission.

D'autre part, l'utilisation pour l'alimentation des chaudièrés de l'eau distillée et absolument pure, provenant de la condensation des turbines, est une très précieuse faculté.

On a été ainsi conduit, dans la plupart des cas et sauf d rares exceptions, à l'emploi de condenseurs à surface du type à contre-courants desservis au moyen de pompes à air sec ou de pompes à air humide (généralement du ty pe Ed'wards).

La surface réfrigérante des condenseurs est généralement prise de $0 \mathrm{mc} 25$ à $0 \mathrm{mq} 35$ par kilowatt de puissance, et quant au débit le plus favorable pour l'eau de circula' tion, il dépend des conditions dans lesquelles elle est obtenue, c'est-à-dire suivant qu'elle doit être puisée à plus ou moins grande profondeur, et suivant qu'elle est empruntée à une source d'eau courante ou à un réfrigérant.

Toutefois, ce débit ne tombe guére au-dessous de 350 litres par kilowatt et il est souvent poussé jusqu'à 600 litres.

Nous devons ajouter que depuis l'apparition des turbines à vapeur, de grands progrès ont été apportés à l'établissement des appareils de condensation, et il est probable qu'ils s'accentueront encore dans l'avenir.

Les hautes pressions d'admission et une surchauffe notable sont favorables au fonctionnement des turbines à vapeur, comme ils le sont pour celui des machines à vapeur (quoique, peut-être, à un degré un peu moins marqué).

(t) Avec les machines à vapeur on sait qu'il est pratiquement impossible d'utıliser cette détente totale jusqu'a un vide de 93 à $940 / 0$, car on arriverant à des dimensions de cylindres inacceptables, doù résulteralent un prix très élevé et des pertes par frottement exagérées.

Dans les turbines, au contraire, le volume de vapeur admis n'inter-

vient que pour les sections d'orifice, et on peut pousser la detente et le

vide aussi loin que possiblo en utilisant d'une manière efficace l'énergie correspondant à cette détente.
Les essais faits sur les differents fypes de turbines ont permis de constater une économie à peu près uniforme de 1,5 à $2 \%$ par dix degrés centigrades de surchaulfe.

En tenant compte de tous les éléments en présence, un surchauffe à l'admission de la turbine de 75 à 100 degré 3 centigrades paralt constituer les conditions de fonctionnement les plus avantageuses.

Celte surchauffe n'offre, d'ailleurs, aucun inconvéuicnt par suite de la suppression de tout graissage intérieur ot, pour les turbines à action du moins, elle ne donne pas lieu difficultés du fait de la dilatation.

\section{L'Accumulateur Mouterde}

L'accumulateur est un réservoir d'électricité ; il est d'une absolue nécessité dans la plupart des applications électriques : Mais cet appareil, malgré sa simplicité appaınt: était hérissé de difficultés d'ordre physique, chimique et mécanique.

La nécessité des accumulateurs explique les nombreuses tentatives qui ont été faites pour arriver à un appareil pratique et bon marché.

La plupart des accumulateurs existant sont calqués sur la vieille disposition à plaques ; mais l'expérience a démontré

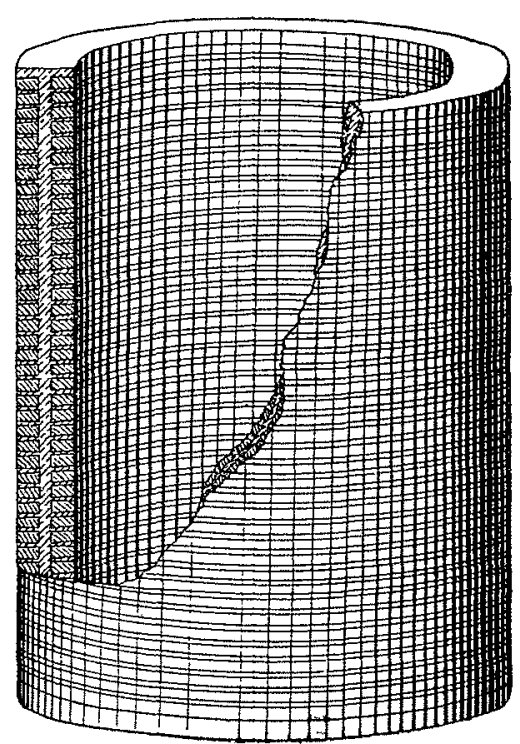

FIG. I. que, sous cette forme de plaques. les électrodestendent toujours à se gauchir et à se mettre en faux contact les unes avec les autres, ce qui amène la perte de l'appareil. D'autre part, la disposition à plaques entraîne l'obligation d'un vaisseau, ou bac en verre, pour contenir l'électrolyte, et aussi quantité d'accessoires en verre, tubes, séparateurs, etc. Le prix de l'accumulateur est de ce fait surchargé par l'obligation de ces accessoires fragiles, coûteux, encombrants et inutiles au fonctionnement réel de l'appareil.

Mais, de ce que les accumulateurs à plaques n'ont pas donné les bons résultats que l'on en attendait, il ne faut pas déduire de cela que le problème soit insoluble; l'expérience a clairement démontré les défauts qu'il fallait supprimer et les points qu'il fallait perfectionner.

Nous donnons ci-après la description d'un nouveau système d'accumulateur dans lequel la disposition à plaques est supprimée : les électrodes sont formées par des surfaces cylindriques concentriques. Ce nouveau système présente des garanties incontestables de solidité, d'endurance et de bon rendement, qui manquent aux anciennes plaques,

Nous remarquons aussi, dans cet appareil, la suppression de tous les accessoires en verre qui grèvent le prix des accumulateurs à plaques.

Les praticiens connaisseurs apprécieront le bien fondé des principes qui ont servi de base à l'étude et à laconstruction de cet appareil et reconnaîtront les avantages exceptionnels qu'il représente comparativement à leurs devancieos. 
Les électrodes qui composent l'accumulateur "Mouterde " sont formés de cylindres rainurés sur leur deux faces, extérieure et intérieure, représentant en quelque sorte une vis-écroul.

Sous cette forme tubulaire, les électrodes sont absolument rigides et indéformables. Chacun, d'ailleurs, sait qu'un tube à paroi même très mince est doué néanmoins d'une très grande rigidité, tandis qu'une plaque même très épaisse pliera sous un effort assez faible.

Dans le cylindre Mouterde, les rainures, dans lesquelles la matière active est encastrée, sont formées par des nervures en queue d'aronde. La pâte active une fois encastrée dans ces filets à orifice resserré, s'y durcit et ne peut plus sortir : elle y est scellée.

La rigidité d'un tube et la soliditéd'un scellement, voilà les principes qui sont

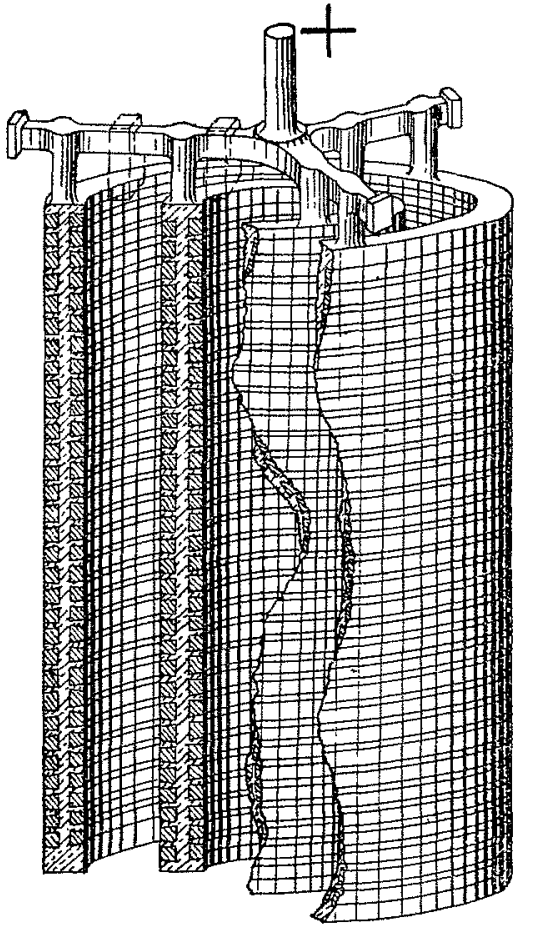

FIG. 2. mis en œuvre dans l'accumulateur Mouterde. Ces principes qui sont de toute évidence, n'ont pas besoin d'être développés plus amplement pour être compris par tout le monde.

Un élément de l'accumulateur Mouterde est formé par l'assemblage d'un certain nombre de cylindres-électrodes groupés en deux séries. Les figures 2 et 3 ci-jointes représentent les deux séries séparées d'un accumulateur composé, par exemple, de cinq cylindres.

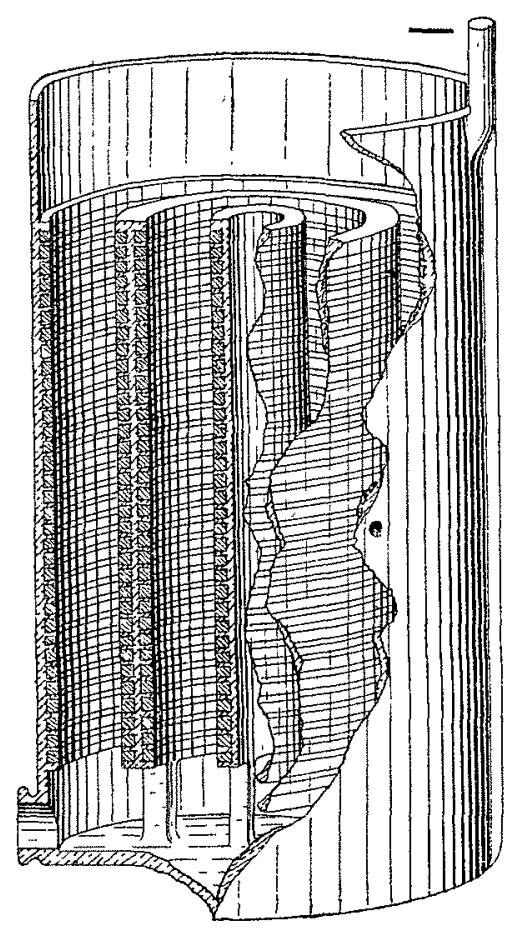

FIG. 3 .
Deux des cylindres sont soudés à un croisillon étoile et forment la série des élcctrodes de signe + , représentée figure 2, D'autre part, les trois autres cylindres sont fixés par leur base sur un disque en plomb, et constituent la série des électrodes de signe-, représentée figure 3 .

Le plus grand des cylindres, muni d'un rebord à la partie supérieure et soudé sur le fond, sert en même temps d'électrode et de récipient pour contenir le liquide. C'est ainsi qu'ont été supprimés le bac en verre et tous les accessoires devenus désormais inutiles.

Au montage, les divers cylindres électrodes de signe + , portés par le croisillon, dont les extrémités sont coiffées de bonnets isolants, viennent s'intercaler entre les divers cylindres électrodes de la série de signe - La figure 4 représente en coupe l'élément complètement monté.
On remarquera que la série des électrodes de signe + , portée par le croisillon, est amovible et interchangeable, de sorte qu'après un usage de plusieurs années, quand elle sera en partie usée par l'action chimique de l'acide, on pourra sans difficulté, et en un instant, la remplacer par une série d'électrodes neuves, sans avoir à toucher à la série des électrodes de signe - , qui, elle, n'est sujette à aucune usure et qui constitue la partie la plus importante de l'appareil,

On remarquera aussi que le croisillon qui réunit toutes les électrodes de signe + porte des goussets isolants qui reposent sur les cylindres de signe -, de sorte que la série + est supportée en un grand nombre de points, assurant ainsi une stabilité parfaite de l'appareil, tout en laissant aux cylindres la liberté de se dilater et de s'allonger.

On remarquera encore le trou de vidange ; ce détail, qui paraît banal, a pourtant une grande importance : il tacilite le nettoyage de l'appareil, nettoyage qui, dans les accumulateurs à plaques, entraîne le démontage complet, et parfois la perte de la batterie.

Les électrodes cylindriques étant indéformables, on a pu les rapprocher jusqu'à ne laisser entre elles que l'espace rigoureusement nécessaire à l'électro. lyte; de ce fait, la résistance intérieure de l'élément est considérablement réduite, et la multiplicité des prises de courant sur les cylindres assure une répartition uniforme du courant sur toutes les surfaces. Le rendement atteint le maximum possible.

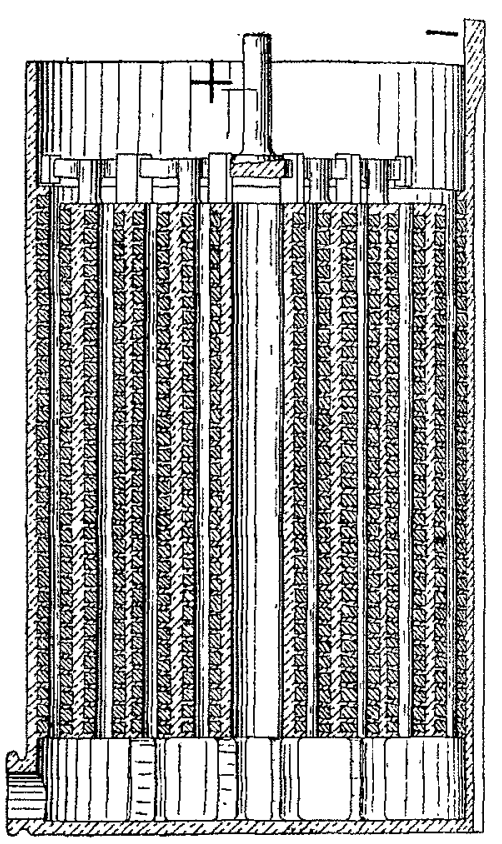

FIG. 4 .
Les accumulateurs Mouterde sont doués d'une solidité et d'une endurance sans limite ; on peut impunément et sans risque d'avarie, les charger et les décharger à des régimes qui détruiraient infailliblement tous les autres sys= tèmes; ils ne nécessitent qu'un entretien très simple: assurer tous les mois le niveau du liquide, et des soins vulgaires de propreté : c'est tout... On peut donc désormais aborder et réaliser à peu de frais toutes les applications de l'électricité qui comportent des accumulateurs : éclairage, transport, emmagasinement et régularisation de la force, stations électriques automatiques, utilisation complète de la force des chutes d'eau accumulée pendant la nuit pour être utilisée pendant le jour ; la traction électrique, l'éclairage des wagons de chemin de fer, la régularisation du coutant des tramways, etc., etc.

C. L.

\section{PRIX ÉMILE DOLLFUS}

La Société Industrielle de Mulhouse a décerné, cette année, le grand prix décennal établı par kmile DoLLFus pour récompenser une grande découverte, invention, ou applicalion, fatte dans les dix dernières années (Médaille d'honneur et 5.000 francs). La Société l'a attrıbué, conjointement, à M. C. A. PArsons, le créateur' du système de turbine à vapeur qui porte ce nom, el à MM. Browì Boveri et $\mathrm{C}^{1 e}$, qui ont construit el perfectionné ce genre de lurbines. 